\title{
Electric-Field Control of Spin-Polarization and Semiconductor-to-Metal Transition in Carbon- Atom-Chain Devices
}

Renato Batista dos Santos, Fernando de Brito Mota, Roberto Rivelino and Gueorgui Kostov Gueorguiev

The self-archived postprint version of this journal article is available at Linköping University Institutional Repository (DiVA):

http:// urn.kb.se/ resolve?urn=urn:nbn:se:liu:diva- 143634

N.B.: When citing this work, cite the original publication.

Batista dos Santos, R., de Brito Mota, F., Rivelino, R., Gueorguiev, G. K., (2017), Electric-Field Control of Spin-Polarization and Semiconductor-to-Metal Transition in Carbon-Atom-Chain Devices, The J ournal of Physical Chemistry C, 121(46), 26125-26132. https:// doi.org/ 10.1021/ acs.jpcc.7b09447

Original publication available at:

https:// doi.org/ 10.1021/acs.jpcc.7b09447

Copyright: AMER CHEMICAL SOC

Publisher URL Missing 


\section{Electric-Field Control of Spin-Polarization and Semiconductor-to-Metal Transition in Carbon- Atom-Chain Devices}

Renato Batista dos Santos ${ }^{\dagger, \S}$, Fernando de Brito Mota ${ }^{\dagger}$, Roberto Rivelino, ${ }^{*, \dagger}$ and Gueorgui K. Gueorguiev*,‡

${ }^{\dagger}$ Instituto de Física, Universidade Federal da Bahia, 40210-340 Salvador, Bahia, Brazil

$\S$ Instituto Federal Baiano, Campus Bom Jesus da Lapa, 47600-000, Bom Jesus da Lapa, Bahia, Brazil

‡ Department of Physics, Chemistry and Biology (IFM), Linköping University, 58183 Linköping, Sweden 
ABSTRACT. We propose hybrid molecular systems containing small carbon atomic chains interconnected by graphene-like flakes, theoretically predicted as true energy minima, as low-dimensional structures that may be useful in electronic devices at the limit of the atomic miniaturization. The effects of an external electric field applied along the direction of the carbon chains indicate that it is possible to control energy gap and spinpolarization with sufficiently high strength, within the limit of the structural restoring of the systems. In this sense, by applying electric fields with magnitudes in the $1-5 \mathrm{~V} / \mathrm{nm}$ range, we obtain semiconductor-to-metallic transitions for all odd-numbered carbonchain systems proposed here. Furthermore, high-spin-to-low-spin transitions are determined for these systems as a function of the electric field magnitude. In the case of the even-numbered carbon-chain systems, the overall electric field effect is pushing electron density near the Fermi level, leading to a gapless or metallic regime at $3.0 \mathrm{~V} / \mathrm{nm}$. An electric-field control of the spin-polarization of these latter systems is only achieved by doping the extremities of the graphene-like terminations with sulfur atoms. This finding, however, is beneficial for applications of these systems in spin-controlled carbon-based devices connected by gold electrodes, even in the presence of a weak spinorbit coupling. 


\section{INTRODUCTION}

The response of low-dimensional electron systems to an external electric field (EF) plays an underlying role for the development of efficient nanoelectronic devices. ${ }^{1-3}$ In this context, molecular rectifiers have long been the 'holy grail' of molecular electronics. ${ }^{4-6}$ For example, Capozzy et al. ${ }^{7}$ have recently demonstrated that it is possible to obtain single-molecule diodes with high rectification ratios by means of environmental control. Indeed, they induce current rectification in symmetric single-molecule junctions between two gold electrodes breaking the symmetry with an ionic solution. Other interesting issue regarding low-dimensional electron systems is the application of an external EF to generate and control spin-polarization as well as single electron spin. ${ }^{8-10}$ In this case, spin states may be manipulated at low dimensions to yield recoverable spin-based quantum information ${ }^{11}$ controlled by an external EF.

Taking into account the electrical response of a nanostructured system, a perfect candidate to be monolithically integrated in a nanocircuit might gather tunable electronic properties, size-scalability, beyond structural stability and robustness against external electric fields. ${ }^{12-15}$ At the atomic level, a one-dimensional (1D) nanowire device represents the ultimate limit in nanoelectronics. ${ }^{6,16}$ Hence, by considering distinct stable atomic chains, ${ }^{18-24}$ one may attain small and robust circuit elements integrated with other electronic components. ${ }^{25,26}$ In this direction, 1D carbon chains may play a central role in miniaturization of electronic devices. ${ }^{27,28}$ Several theoretical studies have pointed out that the electrical response of $s p$-hybridized carbon chains may exhibit high conductivity,

depending on both number and parity of carbon atoms in the string. ${ }^{29-32}$ Experimentally, ${ }^{33}$ however, the conductance of 1D carbon chains appears to be an order of magnitude lower than theoretical predictions. 
Such studies have also indicated that chains containing an even number of carbon atoms have higher conductivity, whereas those containing an odd number of carbon atoms possess a lower resistance. ${ }^{29}$ Yet, the electronic properties of carbon atomic chains strongly depend on the string length, nature of terminal junctions, and relative orientation of the terminal groups interconnecting the chain. ${ }^{34}$ Thus, metallic or semiconducting channels made of $1 \mathrm{D}$ carbon chains can be achieved by controlling several types of variables in the low-dimensional devices. It is usually possible breaking the electrical symmetry by changing the chemical nature of one of the two terminations or by applying an external EF parallel to the chains. This latter option is of our interest here because of important physical reasons ${ }^{35}$ that we will discuss in the following.

In this paper, we consider low-dimensional systems containing carbon-atom chains and investigate how finite external electric fields parallel to the chains (in the limit of restoring structural changes) ${ }^{36}$ may control the density of electron states near the Fermi level and spin states as well. Our study is based on density functional theory (DFT) computational simulations $s^{37,38}$ and the proposed molecular devices are obtained as structurally stable systems. Based on the electronic behavior of other carbon-atomic-wire systems, ${ }^{22,23}$ we have selected chains containing an even/odd number of carbon atoms. Furthermore, for systems interconnected by a single carbon chain, we have analyzed the EF effects on sulfur-containing terminations (substituting $\mathrm{H}$ by $\mathrm{S}$ atoms at the direction of the carbon chain), since sulfur is known as an important contact for gold electrodes ${ }^{39}$ and easily binds to polycyclic aromatic hydrocarbon. ${ }^{40}$ For this reason, we have also considered the EF effects on single carbon atomic chains between terminations containing S-Au bonds. 


\section{COMPUTATIONAL METHODS}

Our proposed molecular devices consist of two-dimensional graphene-flakes (ovalene molecules) interconnected by one, two, and three linear carbon chains, containing five and six carbon atoms. Additionally, we have considered single-chain systems doped with sulfur atoms in the extremities, and with S-Au bonds, as displayed in Figure 1. These structures have been firstly fully-optimized with the B3LYP hybrid functional combined with the 6-31G(d,p) basis set for H, C, and S atoms, and with LANL2 ECP/basis set for $\mathrm{Au}$ atoms, as implemented in the Gaussian 03 program. ${ }^{37}$ The B3LYP/6-31G(d,p) level of theory has been successfully employed to addressing structural and electronic properties of similar systems, ${ }^{22,23,41}$ and has been found as the best compromise between computational cost for larger systems and accuracy. Furthermore, vibrational frequency calculations, within the harmonic approximation, were carried out for all these systems. All vibrational spectra indicate that they converge to true energy minima. The lowest calculated frequency for each system is reported in Table S1 in the Supporting Information.

To investigate the effects of sufficiently high ${ }^{36}$ external EF on these systems, we have employed the SIESTA program. ${ }^{38}$ In this case, we perform DFT calculations in the presence of a finite EF by solving the standard Kohn-Sham (KS) equations, with normconserving pseudopotentials and double- $\zeta$ basis sets including polarization functions, at the $\Gamma$ point of the Brillouin zone. For the exchange-correlation potential, the generalized gradient approximation given by Perdew-Burke-Ernzerhof has been employed. This procedure has been proven to be successful in predicting magnetic states in graphene quantum dots, ${ }^{42}$ as well as for electron transport through graphene-carbon-chain junctions, ${ }^{43}$ in the presence of uniform electric fields. During the process of geometry 
relaxation, under external electric fields with magnitudes in the range of $\left|\vec{E}_{\text {ext }}\right|=0.0-5.0$ $\mathrm{V} / \mathrm{nm}$, the constituent atoms are allowed to relax until forces decrease below $10^{-3} \mathrm{eV} / \AA$. The total energy convergence criterion adopted was of $10^{-5} \mathrm{eV}$. The optimized structures were obtained sequentially by increasing of the EF magnitude. For example, the initial structure, when $\left|\vec{E}_{\text {ext }}\right|=1.0 \mathrm{~V} / \mathrm{nm}$, was considered from the optimized structure obtained when $\left|\vec{E}_{\text {ext }}\right|=0.0 \mathrm{~V} / \mathrm{nm}$. When $\left|\vec{E}_{\text {ext }}\right|=2.0 \mathrm{~V} / \mathrm{nm}$, we have considered as initial structure that obtained at $\left|\vec{E}_{\text {ext }}\right|=1.0 \mathrm{~V} / \mathrm{nm}$, and thus up to $5.0 \mathrm{~V} / \mathrm{nm}$. Hence, the spin excess of each structure is calculated after both electron densities and geometries achieve the convergence criteria (see Figures S1 and S2 the Supporting Information). 
(a)

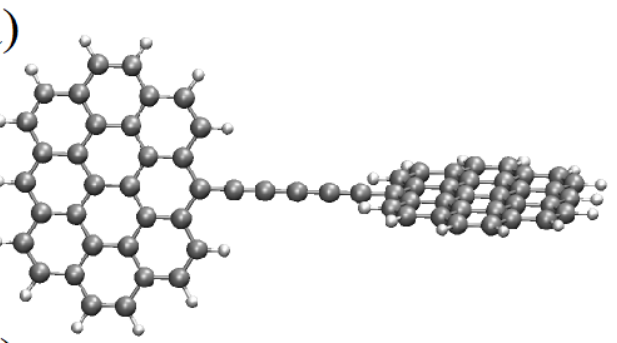

(c)

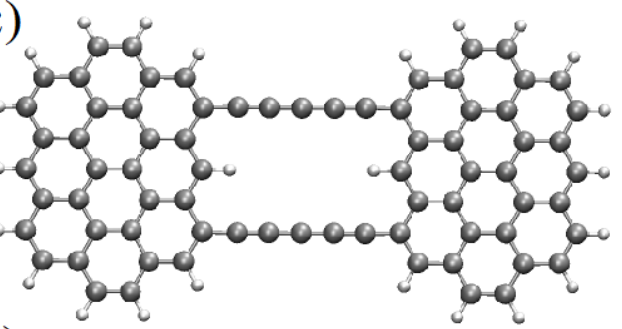

(e)

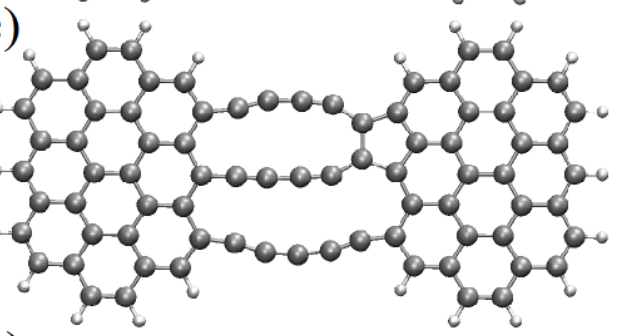

(g)

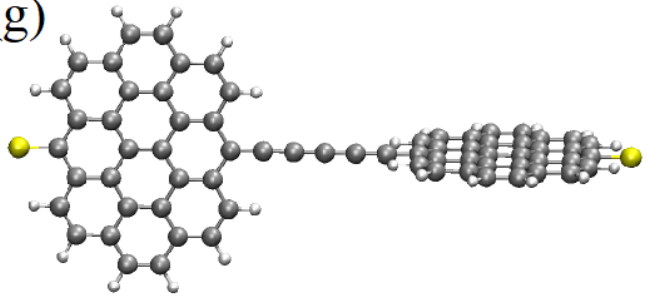

(i)

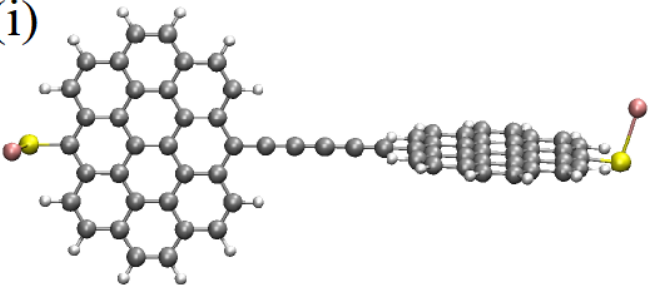

(b)

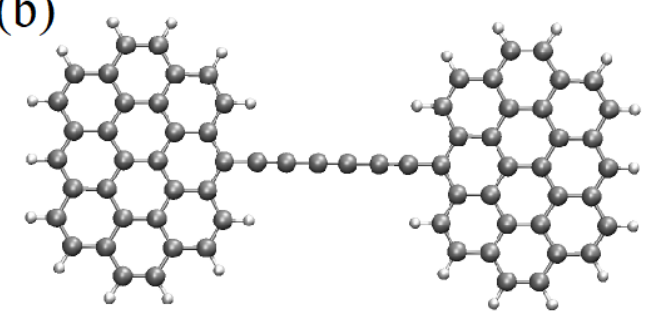

(d)

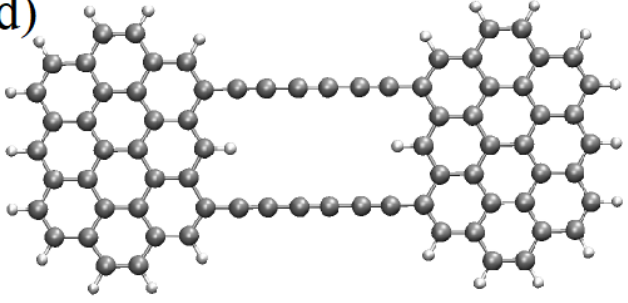

(f)

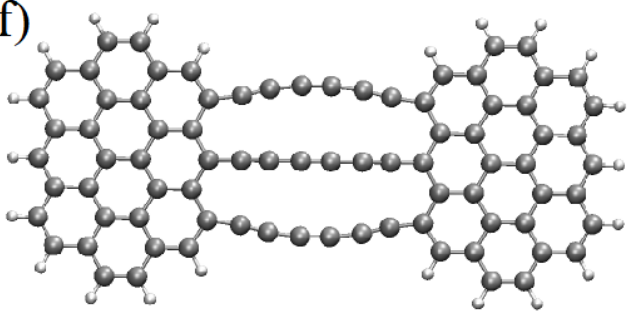

(h)

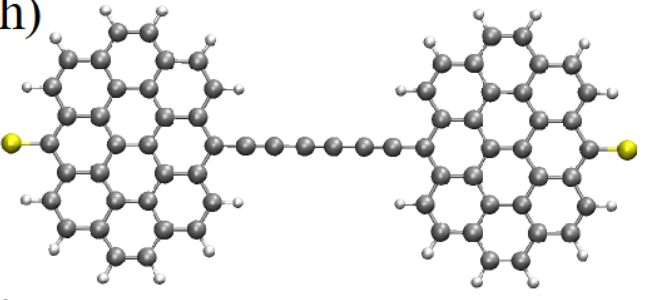

(j)

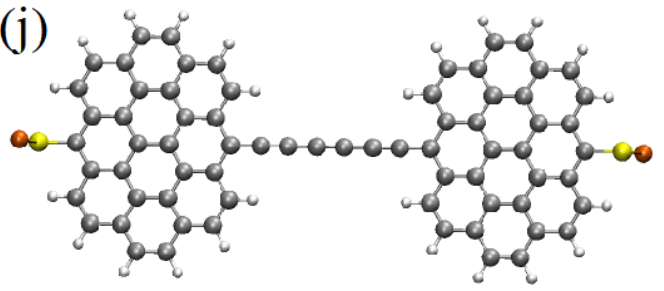

Figure 1. Optimized structures of the proposed low-dimensional systems at the B3LYP/6-31G(d,p) level of theory: (a)-(j). A torsional angle between the two ovalene molecules of $83.7^{\circ}$ is found for the single-chain 5-CAC system (a), whereas for the singlechain 6-CAC system (b) the two ovalene molecules are in the same plane. For the case of the triple 5-chain system (e), a pentagonal reconstruction is found in one side of the contacts to the ovalene molecule.

\section{RESULTS AND DISCUSSION}

Our simulations predict that it is possible tuning a semiconductor-to-metal transition, as well as a spin-polarization in the systems proposed in Figure 1, by setting an appropriate 
magnitude for the external EF. We display the calculated spin-polarized partial density of states (PDOS) in Figure S1 for the optimized graphene-like flakes interconnecting one, two, and three atomic chains containing five carbon atoms (5-CAC), in the $1-5 \mathrm{~V} / \mathrm{nm}$ range. Figure 2 summarizes the HOMO-LUMO energy gap as a function of the magnitude of the applied EF. As seen, in the absence of an external EF, all these systems are gaped, exhibiting an insulator-semiconducting character, in agreement with the electronic structure of polyynes. ${ }^{32}$ By turning on the EF magnitude to $1.0 \mathrm{~V} / \mathrm{nm}$, we find a semiconductor-to-metal transition for the systems that contain one and three 5-CAC, while for the system containing two parallel 5-CAC the energy gap is only reduced by a small amount. Notwithstanding, as we will discuss in the following, the spin-polarization of 5-CAC systems is significantly altered in the presence of an EF. Overall, for field magnitudes in the range of $2-3 \mathrm{~V} / \mathrm{nm}$, all these three systems achieve a metallic character. Interestingly, at 4-6 V/nm the single-chain system is again insulating, while double- and triple-chain systems continue to be metallic.

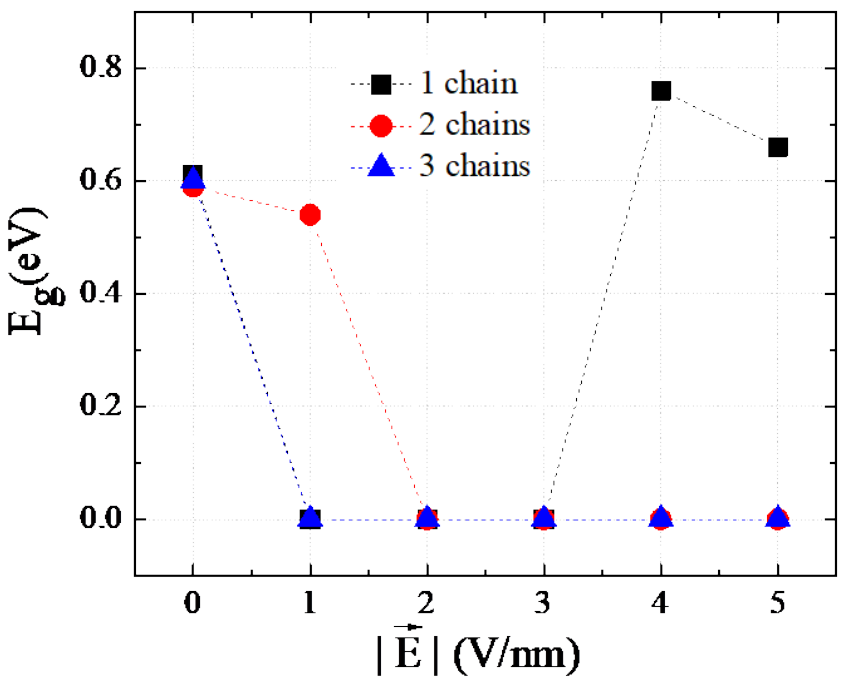

Figure 2. HOMO-LUMO energy gap $\left(E_{\mathrm{g}}\right)$ of the 5-CAC systems as a function of the magnitude of the external EF $(|\vec{E}|)$ applied parallel to the chains (see Figure S1). 
It is well known that the electrical conduction of single chains can exhibit a type of oscillatory pattern as a function of the number of carbon atom, ${ }^{29,44}$ as a result of the interaction with the contact. Here, we show that in the case of a single 5-CAC system the density of electron states may oscillate between insulating and metallic regimes, depending on the strength of the applied EF (see also Figure S1). In this case, the EF effect is inducing electron density from the contact to the chain, giving rise to an alternate polyyne-cumulene-polyyne pattern in the electronic structure of the chain. As displayed in Figure 2, at zero field, our calculated energy gap is $0.61 \mathrm{eV}$, which may completely vanish between 1-2 V/nm, and starts to open a gap from $3.0 \mathrm{~V} / \mathrm{nm}$, reaching energies of $0.76 \mathrm{eV}$ and $0.66 \mathrm{eV}$ for applied EF magnitude of $4.0 \mathrm{~V} / \mathrm{nm}$ and $5.0 \mathrm{~V} / \mathrm{nm}$, respectively. However, for double and triple 5-CAC systems, which have more than one conductive channel, the overall effect of the external EF is moving electron density to the parallel chains, producing a semiconducting-to-metallic behavior and keeping the systems with a metallic character for higher fields. It is important to mention that in the case of the double-chain system an energy gap persists even under an electric field of $1.0 \mathrm{~V} / \mathrm{nm}$, before reaching the transition to a metallic system.

The calculated trend of the spin-polarization of the 5-CAC systems as a function of the EF magnitude is displayed in Figure 3. The spin multiplicity of the low-lying energy ground states of these systems is obtained in the absence/presence of an external EF by optimizing the geometries in each situation with the PBE scheme (see additional information in Figure S1). This means that the electron density is also completely relaxed in the presence of EF. At this level of calculation, in the absence of EF, the relaxed structure of the double 5-CAC system converges to $4 \mu_{B}$ (i.e., it is a spin-quintet state), whereas the relaxed structures of single and triple 5-CAC converge to $2 \mu_{B}$ (i.e., it is a spin-triplet state). In Figure 4, we display the spin density difference between spin-up and 
spin-down for the single and double 5-CAC systems. Indeed, determining spin states is very sensitive to the choice of the exchange-correlation functional; ${ }^{45}$ however, this dependence on spin does not disable the EF effect on the multiplicity of the system. Furthermore, high-spin systems can be experimentally prepared and are of great interest towards molecular magnets, and their possible applications as building blocks for materials exhibiting magnetic ordering, ${ }^{46}$ as well as in spin filtering ${ }^{47,48}$ and spintronic applications. ${ }^{49}$

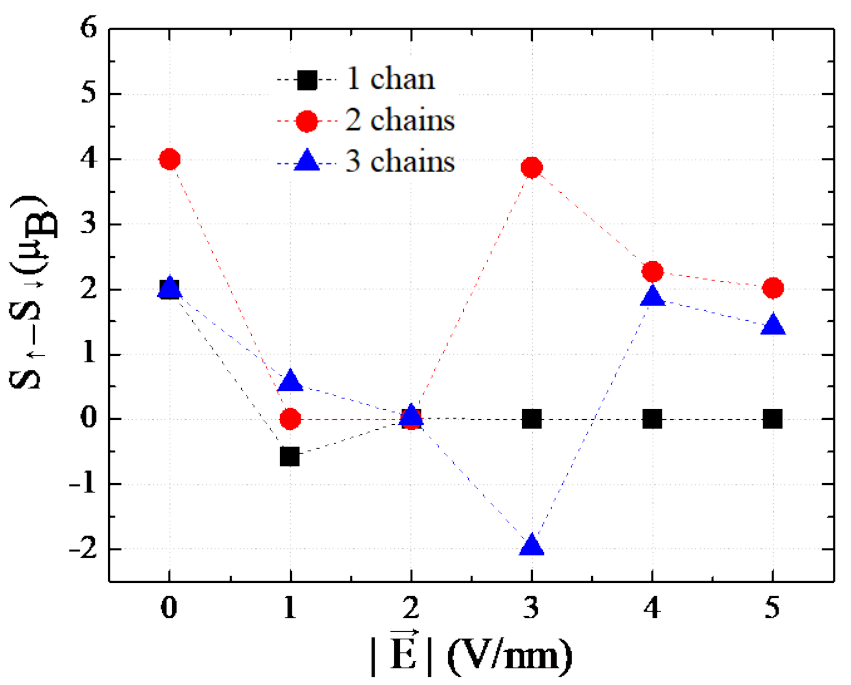

Figure 3. Spin-polarization in terms of the difference between spin-up and spin-down of the 5-CAC systems as a function of the magnitude of the external EF applied parallel to the chains (see Figure S1). 

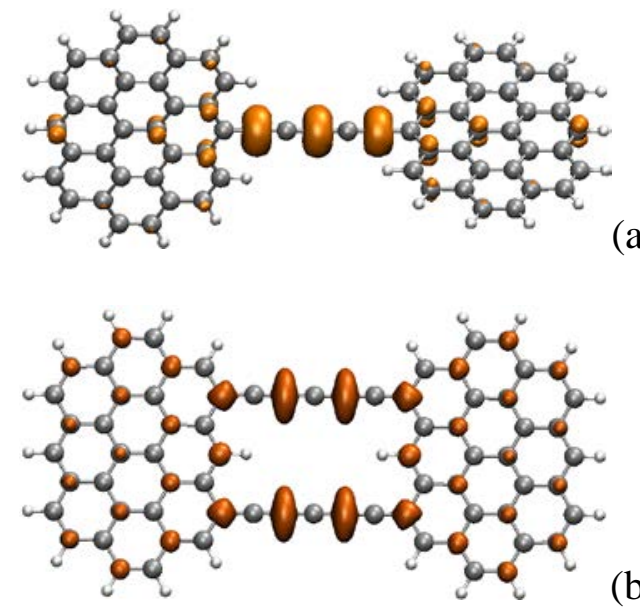

(a)

Figure 4. Spin density difference, $\rho(\uparrow)-\rho(\downarrow)$, of (a) the single 5-CAC system in its spintriplet state and (b) the double 5-CAC system in its spin-quintet state.

Here, we give evidence that an external EF can control the spin-polarization and, consequently, the spin multiplicity transitions in these systems connecting odd-numbered carbon atomic chains. Upon turning on the EF magnitude for $1.0 \mathrm{~V} / \mathrm{nm}$, we notice a strong reduction in the spin multiplicity of the systems. For example, the double 5-CAC system undergoes a quintet-singlet transition mediated by electric fields with magnitudes of 1.0 $\mathrm{V} / \mathrm{nm}$ and $2.0 \mathrm{~V} / \mathrm{nm}$. However, at $3.0 \mathrm{~V} / \mathrm{nm}$, the spin multiplicity of this system changes again to a spin-quintet state, converging to a spin-triplet state at $5.0 \mathrm{~V} / \mathrm{nm}$. Conversely, the single 5-CAC system appears to be more robust at high electric fields. Its spin multiplicity is reduced at $1.0 \mathrm{~V} / \mathrm{nm}$ and it remains as a spin-singlet state in electric fields as high as $5.0 \mathrm{~V} / \mathrm{nm}$. Among these three molecular devices, the triple 5-CAC system experiences drastic oscillations in the spin-polarization under external electric fields, although it behaves as a metallic system from $1.0 \mathrm{~V} / \mathrm{nm}$ (see Figure 2). For this case, we notice (i) a spin excess reduction to $0.58 \mu_{B}$ at $1.0 \mathrm{~V} / \mathrm{nm}$; (ii) a triplet-singlet transition at 2.0 V/nm; (iii) a spin-density inversion between spin-up and spin-down distributions at 3.0 V/nm; (iv) a spin excess increase to $1.86 \mu_{B}$ at $4.0 \mathrm{~V} / \mathrm{nm}$; and (v) a spin excess decrease to $1.42 \mu_{B}$ at $5.0 \mathrm{~V} / \mathrm{nm}$. 
In addition to tuning the energy gap of the 5-CAC systems, other important EF effect noticed here is to control their spin states. Clearly, these results may be of interest to read and store quantum information by spin transfer from spin-polarized currents, with the use of an EF. As an example, we analyze more deeply the double-chain system, which is gapped at an EF with magnitude of $1.0 \mathrm{~V} / \mathrm{nm}$ with a spin-singlet state (see Figure 1c and Figure 3). By increasing the field strength to $2.0 \mathrm{~V} / \mathrm{nm}$, this system becomes gapless and continues to be a spin-singlet. However, by increasing the strength to $3.0 \mathrm{~V} / \mathrm{nm}$ the system becomes metallic and achieves a high-spin state. Finally, at $5.0 \mathrm{~V} / \mathrm{nm}$, the doublechain system converges to a spin-triplet state. In this sense, we show that tuning an appropriate EF is useful to manipulate spin states, even for systems exhibiting a very weak spin-orbit coupling, such as carbon-based structures. ${ }^{50,51}$

In Figure S2, we display the calculated spin-polarized PDOS for the optimized graphene-like flakes interconnecting one, two, and three atomic chains containing six carbon atoms (6-CAC), in the 1-5 V/nm range. For these cases, a semiconductor-to-metal transition is only noticed for single and double 6-CAC systems. The present results show that a triple 6-CAC system does not exhibit a metallic character under electric fields with magnitude as high as $5.0 \mathrm{~V} / \mathrm{nm}$, although the energy gap of this system can be considerably reduced in the presence of an external EF. This behavior may be attributable to the geometric distortion of the two outermost chains without defects formation (see Figure 1f and Figure S2c) of the triple 6-CAC system, originating a hindrance to the charge motion through the chains during the application of the EF. We will discuss this issue with more details in the following.

Differently from the three 5-CAC systems, the ground states obtained for the relaxed 6-CAC corresponding systems converge to low-spin states; i.e., all of them are singlet-spin states in the absence of an external EF. Moreover, their HOMO-LUMO 
energy gaps are a little higher than the corresponding values of the 5-CAC systems. We report the energy gap as a function of the EF magnitude the 6-CAC systems in Figure 5. The main effect of turning on an external EF in these molecular devices is reducing their energy gaps, but conserving their singlet-spin states. From $1.0 \mathrm{~V} / \mathrm{nm}$ up to $5.0 \mathrm{~V} / \mathrm{nm}$, the EF is not able to generate a spin-polarization in these systems. For example, at $1.0 \mathrm{~V} / \mathrm{nm}$, the energy gap of the single 6-CAC system is reduced from $1.01 \mathrm{eV}$ (at zero field) to 0.62 $\mathrm{eV}$, keeping its total spin as zero. By increasing the EF magnitude to $2.0 \mathrm{~V} / \mathrm{nm}$, the energy gap reduces to $0.28 \mathrm{eV}$ and, at $3.0 \mathrm{~V} / \mathrm{nm}$, the system becomes gapless or narrow-gapped, still conserving the spin-singlet state. A very similar behavior is also observed for the double 6-CAC system, which also achieves a metallic character for an EF magnitude starting from $3.0 \mathrm{~V} / \mathrm{nm}$, blocking the spin-polarization for electrons passing through these chains.

It is particularly interesting to detail here the electrical response of the triple 6CAC system, which continues to be gapped for electric fields with magnitude up to 5.0 $\mathrm{V} / \mathrm{nm}$. At zero field, this system exhibits an energy gap of $0.66 \mathrm{eV}$, which is reduced to 0.56 at $1.0 \mathrm{~V} / \mathrm{nm}$ and to $0.43 \mathrm{eV}$ at $2.0 \mathrm{~V} / \mathrm{nm}$, achieving a smaller value of $0.34 \mathrm{eV}$ at 4.0 $\mathrm{V} / \mathrm{nm}$ and $5.0 \mathrm{~V} / \mathrm{nm}$. As displayed in Figure 1f, the two outermost chains of this system undergo a curvature, whereas the central chain remains linear, after full geometry optimization of this system, resulting in a totally symmetric structure. This spatial distortion is a consequence of the electric repulsion between the central chain and its neighbors and leads to a perturbation in the $\pi$-electron delocalization. In the case of the triple 5-CAC system, there is also a curvature in the chains, but exhibiting a pentagonal reconstruction in one of the graphene-like terminations upon geometry optimization. This defect, which is typical in graphene constriction with short carbon chains, ${ }^{52}$ breaks both the structural and electrical symmetry of the system. For this reason, on the contrary to 
the triple 6-CAC system case, its related 5-CAC system undergoes more easily a semiconductor-to-metal transition tuned by an EF.

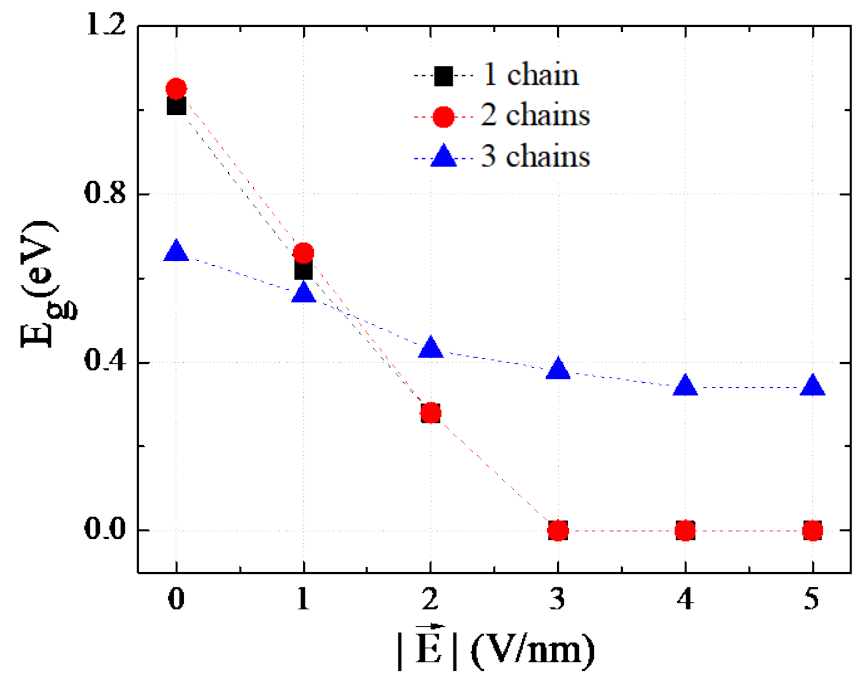

Figure 5. HOMO-LUMO energy gap $\left(E_{\mathrm{g}}\right)$ of the 6-CAC systems as a function of the magnitude of the external EF ( $|\vec{E}|$ ) applied parallel to the chains (see Figure S2).

In many electronic applications involving carbon-based low-dimensional devices, such as those proposed here, the interaction with metallic electrodes becomes extremely important, since the leads can also control the electronic properties of the system. Usually a sulfur-gold interface ${ }^{53}$ is preferable at the atomic level because of the chemical affinity of these elements and conductivity increase $e^{54-57}$ in the systems. To verify the electrical response of these single 5-CAC and 6-CAC systems as potential candidates in singlemolecule junctions with gold electrodes, we first consider single-chain systems doped with sulfur; i.e, substituting H/S in the graphene-like flakes (ovalene molecules) along the direction of the chains (see Figure 1g, Figure 1h, and Figure 6). As seen, the H/S substitution leads the electronic ground states of both systems to singlet-spin states in the absence of an external EF. Furthermore, as expected, it reduces the HOMO-LUMO energy gap of these systems, as compared to the corresponding undoped structures (see Figures S1 and S2). 


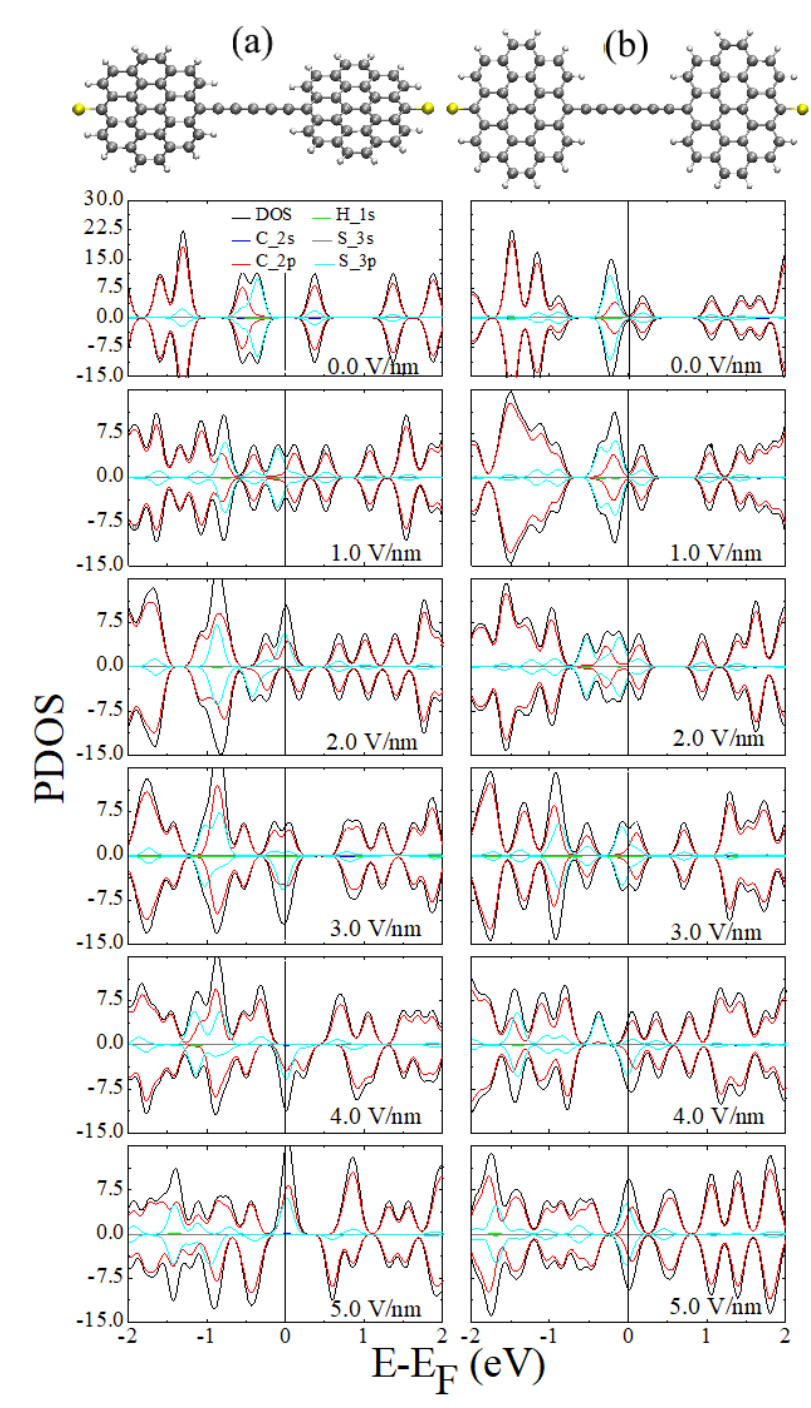

Figure 6. Optimized structures and spin-polarized PDOS of the single S-doped $n$-CAC systems (S-Ø-C $\left.{ }_{n}-\varnothing-S\right), n=5,6$, under external electric fields with magnitude varying from $0-5 \mathrm{~V} / \mathrm{nm}$. (a) $\mathrm{S}-\varnothing-\mathrm{C}_{5}-\varnothing-\mathrm{S}$ and (b) $\mathrm{S}-\varnothing-\mathrm{C}_{6}-\varnothing-\mathrm{S}$ systems. $\mathrm{S}$ atoms forms double bonds with the carbon atoms and are represented by yellow spheres.

At zero field, the pristine single 5-CAC system exhibits an energy gap of $0.61 \mathrm{eV}$ in its spin-triplet state, whereas the corresponding S-doped system exhibits an energy gap of $0.47 \mathrm{eV}$ in its spin-singlet state. For the single 6-CAC system, the S-doping effect is reducing the energy gap from $1.01 \mathrm{eV}$ of the undoped system to zero, but conserving the spin-singlet state. By turning on the EF magnitude for $1.0 \mathrm{~V} / \mathrm{nm}$ on these two systems (S$\varnothing-C_{5}-\varnothing-S$ and $\left.S-\varnothing-C_{6}-\varnothing-S\right)$, we notice that they exhibit metallic character, with density of states crossing the Fermi level, as displayed in Figure 6. The KS-orbitals that 
most contribute to the semiconducting-to-metallic regime are the $2 p$ of carbon and $3 p$ of sulfur. Even increasing the magnitude of the applied EF to $2.0 \mathrm{~V} / \mathrm{nm}$, in the case of the $\mathrm{S}-\varnothing-\mathrm{C}_{5}-\varnothing-\mathrm{S}$, and to $3.0 \mathrm{~V} / \mathrm{nm}$, in the case of $\mathrm{S}-\varnothing-\mathrm{C}_{6}-\varnothing-\mathrm{S}$, these systems maintain the zero spin-polarization. However, at $3.0 \mathrm{~V} / \mathrm{nm}, \mathrm{S}-\varnothing-\mathrm{C}_{5}-\varnothing-\mathrm{S}$ exhibits a residual spin excess of $0.50 \mu_{\mathrm{B}}$ and, at $4.0 \mathrm{~V} / \mathrm{nm}, \mathrm{S}-\varnothing-\mathrm{C}_{6}-\varnothing-\mathrm{S}$ exhibits a smaller residual spin excess of $0.37 \mu_{\mathrm{B}}$. It is important to notice here that only in the case of $S-\varnothing-C_{5}-\varnothing-S$ a singlettriplet transition is found at $4.0 \mathrm{~V} / \mathrm{nm}$, with a spin-polarization inversion at $5.0 \mathrm{~V} / \mathrm{nm}$. For this case, it becomes clear that by setting a proper external EF is possible to generate a spin-polarization in the system, even without considering the spin-orbit coupling effect in the calculations, which may become important upon S-doping of the molecular devices. The EF effects on these two S-doped single-chain systems are summarized in Table 1. 
Table 1. Electric field effect (magnitude in $\mathrm{V} / \mathrm{nm}$ ) on the calculated HOMO-LUMO energy gaps (in $\mathrm{eV}$ ) and spin-polarization (in $\mu_{\mathrm{B}}$ ) of the S-doped (S- $\varnothing-\mathrm{C}_{n}-\varnothing-\mathrm{S}$ ) and covalently bound to gold $\left(\mathrm{Au}-\mathrm{S}-\varnothing-\mathrm{C}_{n}-\varnothing-\mathrm{S}-\mathrm{Au}\right)$ single-chain systems (see PDOS in Figure 6 and Figure 7)

\begin{tabular}{cccccc}
\hline \multirow{2}{*}{$n$} & $|\vec{E}|$ & \multicolumn{2}{c}{$\mathrm{S}-\varnothing-\mathrm{C}_{n}-\varnothing-\mathrm{S}$} & \multicolumn{2}{c}{$\mathrm{Au}-\mathrm{S}-\varnothing-\mathrm{C}_{n}-\varnothing-\mathrm{S}-\mathrm{Au}$} \\
\cline { 3 - 6 } & & $E_{g}$ & $S \uparrow-S \downarrow$ & $E_{g}$ & $S \uparrow-S \downarrow$ \\
\hline \multirow{3}{*}{5} & 0.47 & 0.00 & 0.24 & 2.00 \\
& 0.0 & 0.00 & 0.00 & 0.00 & 1.48 \\
& 1.0 & 0.00 & 0.00 & 0.00 & 0.00 \\
& 2.0 & 0.00 & 0.50 & 0.20 & 0.00 \\
& 3.0 & 0.00 & 2.00 & 0.00 & -0.20 \\
& 4.0 & 0.00 & -2.00 & --- & 0.00 \\
& 5.0 & 0.00 & 0.00 & 0.59 & 0.00 \\
& 0.0 & 0.00 & 0.00 & 0.00 & 0.00 \\
& 1.0 & 0.00 & 0.00 & 0.00 & 0.01 \\
& 2.0 & 0.00 & 0.00 & 0.00 & 0.00 \\
& 3.0 & 0.00 & 0.37 & 0.00 & --- \\
\hline
\end{tabular}

Second, we have considered S-doped $n$-CAC systems $(n=5,6)$ covalently bound to gold atoms as displayed in Figure 7 (see also Figure 1i and Figure 1j). Indeed, this does not mimic the effects of the metallic leads in the system, but it gives a preliminary indication of how orbitals belonging to the Au atoms can affect the electrical response of the systems (see Table 1). For example, when the S-doped single 5-CAC system is bound to $\mathrm{Au}$ atoms in its extremities, resulting in $\mathrm{Au}-\mathrm{S}-\varnothing-\mathrm{C}_{5}-\varnothing-\mathrm{S}-\mathrm{Au}$, the HOMO-LUMO energy gap is reduced to $0.24 \mathrm{eV}$ and converges to a spin-triplet state. Hence, turning on an external EF with magnitude $1.0 \mathrm{~V} / \mathrm{nm}$ leads this system to a metallic regime, exhibiting a spin excess of $1.48 \mu_{\mathrm{B}}$. Interestingly, at $2.0 \mathrm{~V} / \mathrm{nm}$, we find a triplet-singlet transition in this system, keeping its spin-singlet state up to $3.0 \mathrm{~V} / \mathrm{nm}$, although an energy gap is opened with $0.20 \mathrm{eV}$. At $4.0 \mathrm{~V} / \mathrm{nm}$, this system achieves again its metallic character, but 
inverting its spin-polarization with a spin excess of $-0.20 \mu_{\mathrm{B}}$. For magnitude of electric field higher than $4.0 \mathrm{~V} / \mathrm{nm}$ a convergence of the electronic structure is difficult to be obtained for this system. In the case of $\mathrm{Au}-\mathrm{S}-\varnothing-\mathrm{C}_{6}-\varnothing-\mathrm{S}-\mathrm{Au}$, the system exhibits an energy gap of $0.59 \mathrm{eV}$ at zero field in its spin-singlet state. Starting from electric field with magnitude higher than $1.0 \mathrm{~V} / \mathrm{nm}$, this system becomes metallic, but keeps its spinsinglet state (see Table 1).

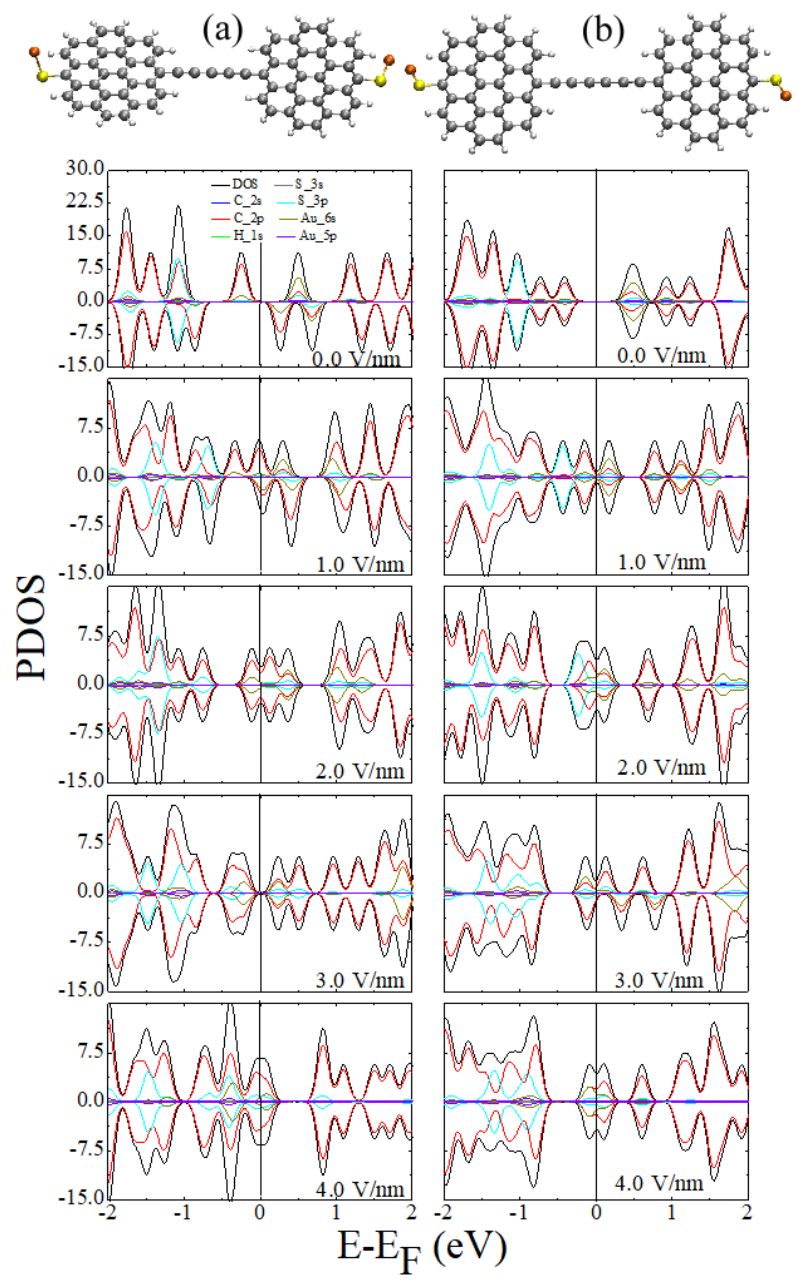

Figure 7. Optimized structures and spin-polarized PDOS of the single S-doped $n$-CAC systems covalently bound to gold atoms ( $\left.\mathrm{Au}-\mathrm{S}-\varnothing-\mathrm{C}_{n}-\varnothing-\mathrm{S}-\mathrm{Au}\right), n=5,6$, under external electric fields with magnitude varying from $0-5 \mathrm{~V} / \mathrm{nm}$. (a) Au-S- $-\mathrm{C}_{5}-\varnothing-\mathrm{S}-\mathrm{Au}$ and (b) $\mathrm{Au}-\mathrm{S}-\varnothing-\mathrm{C}_{6}-\varnothing-\mathrm{S}-\mathrm{Au}$ systems. $\mathrm{S}$ and $\mathrm{Au}$ atoms are respectively represented by yellow and red spheres. 


\section{CONCLUSIONS}

In conclusion, we have investigated how an external EF, in the limit of restoring structural changes in the system, may affect energy gaps and spin-polarizations of single, double, and triple carbon atomic chains interconnected by equal graphene-like flakes. The electric fields are applied parallel to the chains in order to break the electrical symmetry of the proposed low-dimensional devices. The main effect of this electrical perturbation is tuning a semiconductor-to-metal transition in most of the considered and studied systems. An exception is found for the triple 6-CAC system, which keeps its semiconducting character for EF with magnitude as high as $5.0 \mathrm{~V} / \mathrm{nm}$. Interestingly, the single 5-CAC system exhibits an oscillatory electronic behavior, achieving a metallic regime between 1-3 V/nm and a semiconducting regime between 3-5 V/nm, whereas double and triple 5CAC systems appear to exhibit a semiconductor-to-metal transition for well-established EF magnitudes.

Other important effect of the external EF is controlling the spin-polarization of the systems interconnecting odd-numbered carbon atomic chains, even for systems experiencing a mild spin-orbit coupling. In the case of the 5-CAC systems, the EF is able to produce high-spin-to-low-spin or vice-versa transitions. In the case of the single 5CAC system, a triplet-singlet transition occurs for an EF magnitude higher than $1.0 \mathrm{~V} / \mathrm{nm}$. Yet, for the double 5-CAC system, it is found a quintet-singlet transition at $1-2 \mathrm{~V} / \mathrm{nm}$ and a singlet-quintet transition at $3.0 \mathrm{~V} / \mathrm{nm}$. For the triple 5-CAC system, a triplet-singlet transition occurs at $2.0 \mathrm{~V} / \mathrm{nm}$ with spin-polarization inversion at $3.0 \mathrm{~V} / \mathrm{nm}$. Such effects were not noticed for the 6-CAC systems, by considering the same range of EF applied to the chains. For these three cases, the spin state of the system continues to be a singlet and 
the applied electric fields are not able to generate a spin-polarization. This robustness can be modified, however, by doping the graphene-like terminations.

Doping the single $n$-CAC systems $(n=5,6)$ with sulfur atoms reduces their energy gaps and leads them to spin-singlet states at zero field or under low-magnitude electric fields. For EF magnitudes as high as $3.0 \mathrm{~V} / \mathrm{nm}$ it is possible to generate a spin-polarization in the S-doped single 5-CAC system, originating a spin excess of $0.5 \mu_{\mathrm{B}}$. Most interestingly, at $4.0 \mathrm{~V} / \mathrm{nm}$, it is found a singlet-triplet transition and, at $5.0 \mathrm{~V} / \mathrm{nm}$, a complete spin-polarization inversion in this system. This finding reveals that spin states in carbon-atom-chain devices may be manipulated with an external EF. Binding the terminal S atoms to Au atoms, the spin multiplicity changes again to a triplet state at zero field, but converges to a singlet state at $2.0 \mathrm{~V} / \mathrm{nm}$. In this sense, carbon atomic chains may be feasible candidates as spin filters between gold electrodes. Finally, this study provides a theoretical analysis of the EF effects on small carbon atomic chains and rises important questions about energy gap engineering and spin control in low-dimensional systems that deserve further theoretical and experimental investigation.

\section{ASSOCIATED CONTENT}

\section{Supporting Information.}

Computational details; optimized structures; spin-polarized partial densities of states (PDOS); and lowest vibrational frequencies of the proposed systems.

This material is available free of charge via the Internet at http://pubs.acs.org.

\section{AUTHOR INFORMATION}




\section{Corresponding Authors}

*E-mail: rivelino@ufba.br

*E-mail: gekos@ifm.liu.se

\section{ORCID}

Roberto Rivelino: 0000-0003-2679-1640

Gueorgui K. Gueorguiev: 0000-0001-9402-1491

Renato Batista dos Santos: 0000-0001-7062-1628

Fernando de Brito Mota: 0000-0001-9571-8549

\section{Notes}

The authors declare no competing financial interest.

\section{ACKNOWLEDGMENT}

This work is partially supported by the Brazilian Funding Agencies (CNPq and CAPES) and by the Swedish Research Council (VR) through Swedish Research Links project 3482014-4249. VR 621-2013-5818 is also gratefully acknowledged. GKG gratefully acknowledges support by FLAG-ERA JTC 2015 project GRIFONE and by Carl Tryggers Foundation for Scientific Research (CTS 16:165). We have utilized computational resources of the National Supercomputer Centre at Linköping University.

\section{REFERENCES}


(1) Nitzan, A.; Ratner, M. A. Electron Transport in Molecular Wire Junctions. Science 2003, 300, 1384-1389.

(2) Song, H.; Reed, M. A.; Lee, T. Single Molecule Electronic Devices. Adv. Mater. 2011, 23, 1583-1608.

(3) Iida, K.; Nobusada, K. Electric Field Effects on the Electronic Properties of the Silicene-Amine Interface. Phys. Chem. Chem. Phys. 2016, 18, 15639-15644.

(4) Troisi, A.; Ratner, M. A. Molecular Rectification through Electric Field Induced Conformational Changes. J. Am. Chem. Soc. 2002, 124, 14528-14529.

(5) Aviram, A.; Ratner, M. A. Molecular Rectifiers. Chem. Phys. Lett. 1974, 29, 277-283.

(6) Su, T. A.; Neupane, M.; Steigerwald, M. L.; Venkataraman, L.; Nuckolls, C. Chemical Principles of Single-Molecule Electronics. Nature Rev. Mater. 2016, 1, 16002.

(7) Capozzi, B.; Xia, J.; Adak, O.; Dell, E. J.; Liu, Z.-F.; Taylor, J. C.; Neaton, J. B.; Campos, L. M.; Venkataraman, L. Single-Molecule Diodes with High Rectification Ratios through Environmental Control. Nature Nanotech. 2015, 10, 522-527.

(8) Nowack, K. C.; Koppens, F. H. L.; Yu. Nazarov, V.; Vandersypen, L. M. K. Coherent Control of a Single Electron Spin with Electric Fields. Science 2007, 318, 1430-1433.

(9) Zeng, M. G.; Shen, L.; Cai, Y. Q.; Sha, Z. D.; Feng, Y. P. Perfect Spin-Filter and Spin-Valve in Carbon Atomic Chains. Appl. Phys. Lett. 2010, 96, 042104.

(10) M. Luengo-Kovac, M.; Moraes, F. C. D.; Ferreira, G. J.; Ribeiro, A. S. L.; Gusev, G. M.; Bakarov, A. K.; Sih, V.; Hernandez, F. G. G. Gate Control of the Spin Mobility through the Modification of the Spin-Orbit Interaction in Two-Dimensional Systems. Phys. Rev. B 2017, 95, 245315. 
(11) Laucht, A.; Muhonen, J. T.; Mohiyaddin, F. A.; Kalra, R.; Dehollain, J. P.; Freer, S.; Hudson, F. E.; Veldhorst, M.; Rahman, R.; Klimeck, G., et al. Electrically Controlling Single-Spin Qubits in a Continuous Microwave Field. Sci. Adv. 2015, 1, e1500022.

(12) Aragonès, A. C.; Medina, E.; Ferrer-Huerta, M.; Gimeno, N.; Teixidó, M.; Palma, J. L.; Tao, N.; Ugalde, J. M.; Giralt, E.; Díez-Pérez, I., et al. Measuring the SpinPolarization Power of a Single Chiral Molecule. Small 2017, 13, 1602519.

(13) Xiang, L.; Palma, J. L.; Li, Y.; Mujica, V. Ratner, M. A.; Taoa, N. Gate-Controlled Conductance Switching in DNA. Nature Commun. 2017, 8, 14471.

(14) Mujica, V.; Kemp, M.; Roitberg, A.; Ratner, M. Current-Voltage Characteristics of Molecular Wires: Eigenvalue Staircase, Coulomb Blockade, and Rectification. J. Chem. Phys. 1996, 104, 7296-7305.

(15) Mujica, V.; Roitberg, A. E., Ratner, M. Molecular Wire Conductance: Electrostatic Potential Spatial Profile. J. Chem. Phys. 2000, 112, 6834-6839.

(16) Mota, F. B.; Rivelino, R.; Medeiros, P. V. C.; Mascarenhas, A. J. S.; de Castilho, C. M. C. Hybrid Platforms of Graphane-Graphene 2D Structures: Prototypes for Atomically Precise Nanoelectronics. Phys. Chem. Chem. Phys. 2014, 16, 23558-23563.

(17) Pontes, R. B.; da Silva, E. Z.; Fazzio, A.; da Silva, A. J. R. Symmetry Controlled Spin Polarized Conductance in Au Nanowires. J. Am. Chem. Soc. 2008, 130, 9897-9903. (18) Andrade, N. F.; Aguiar, A. L.; Kim, Y. A.; Endo, M.; Freire, P. T. C.; Brunetto, G.; Galvão, D. S.; Dresselhaus, M. S.; Filho, A. G. S. Linear Carbon Chains under HighPressure Conditions. J. Phys. Chem. C, 2015, 119, 10669-10676. 
(19) Fujimori, T.; Morelos-Gómez, A.; Zhu, Z.; Muramatsu, H.; Futamura, R.; Urita, K.; Terrones, M.; Hayashi, T.; Endo, M.; Hong, S. Y., et al. Conducting Linear Chains of Sulphur inside Carbon Nanotubes. Nature Commun. 2013, 4, 2162.

(20) Fujimori, T.; dos Santos, R. B.; Hayashi, T.; Endo, M.; Kaneko, K.; Tománek, D. Formation and Properties of Selenium Double-Helices inside Double-Wall Carbon Nanotubes: Experiment and Theory. ACS Nano 2013, 7, 5607-5613.

(21) Vierimaa, V.; Krasheninnikov, A. V.; Komsa, H.-P. Phosphorene under Electron Beam: from Monolayer to One-Dimensional Chains. Nanoscale 2016, 8, 7949-7957.

(22) Rivelino, R.; dos Santos, R. B.; Mota, F. B.; Gueorguiev, G. K. Conformational Effects on Structure, Electron States, and Raman Scattering Properties of Linear Carbon Chains Terminated by Graphene-Like Pieces. J. Phys. Chem. C 2010, 114, 16367-16372. (23) dos Santos, R. B.; Rivelino, R.; Mota, F. B.; Gueorguiev, G. K. Effects of N Doping on the Electronic Properties of a Small Carbon Atomic Chain with Distinct $\mathrm{sp}^{2}$ Terminations: A First-Principles Study. Phys. Rev. B 2011, 84, 075417.

(24) Rong, Y.; Warner, J. H. Wired Up: Interconnecting Two-Dimensional Materials with One-Dimensional Atomic Chains. ACS Nano 2014, 8, 11907-11912.

(25) Wimmer, M.; Palma, J. L.; Tarakeshwar, P.; Mujica, V. Single-Molecule Conductance through Hydrogen Bonds: The Role of Resonances. J. Phys. Chem. Lett., 2016, 7, 2977-2980.

(26) Liu, M.; Artyukhov, V. I.; Yakobson, B. I. Mechanochemistry of One-Dimensional Boron: Structural and Electronic Transitions. J. Am. Chem. Soc. 2017, 139, 2111-2117.

(27) Jin, C.; Lan, H.; Peng, L.; Suenaga, K.; Iijima, S. Deriving Carbon Atomic Chains from Graphene. Phys. Rev. Lett. 2009, 102, 205501. 
(28) Cretu, O.; Botello-Mendez, A. R.; Janowska, I.; Pham-Huu, C.; Charlier, J.-C.; Banhart, F. Electrical Transport Measured in Atomic Carbon Chains. Nano Lett. 2013, 13, 3487-3493.

(29) Lang, N. D.; Avouris, P. Oscillatory Conductance of Carbon-Atom Wires. Phys. Rev. Lett. 1998, 81, 3515-3518.

(30) Lang, N. D.; Avouris, P. Carbon-Atom Wires: Charge-Transfer Doping, Voltage Drop, and the Effect of Distortions. Phys. Rev. Lett. 2000, 84, 358-361.

(31) Song, B.; Sanvito, S.; Fang, H. Anomalous I-V Curve for Mono-Atomic Carbon Chains. New J. Phys. 2010, 12, 103017.

(32) Milani, A; Tommasini, M.; Barbieri, V.; Lucotti, A.; Russo, V.; Cataldo, F.; Casari,

C. S. Semiconductor-to-Metal Transition in Carbon-Atom Wires Driven by $\mathrm{sp}^{2}$ Conjugated End Groups. J. Phys. Chem. C 2017, 121, 10562-10570.

(33) Yuzvinsky, T. D.; Mickelson, W.; Aloni, S.; Begtrup, G. E.; Kis, A.; Zettl, A. Shrinking a Carbon Nanotube. Nano Lett. 2006, 6, 2718-2722.

(34) Ravagnan, L.; Manini, N.; Cinquanta, E.; Onida, G.; Sangalli, D.; Motta, C.; Devetta, M.; Bordoni, A.; Piseri, P.; Milani, P. Effect of Axial Torsion on sp Carbon Atomic Wires. Phys. Rev. Lett. 2009, 102, 245502.

(35) Novoselov, K. S.; Geim, A. K.; Morozov, S. V.; Jiang, D.; Zhang, Y.; Dubonos, S. V.; Grigorieva, I. V.; Firsov, A. A. Electric Field Effect in Atomically Thin Carbon Films. Science 2004, 306, 666-669.

(36) Lou, L.; Nordlander, P. Carbon Atomic Chains in Strong Electric Fields. Phys. Rev. B. 1996, 54, 16659-16662. 
(37) Frisch, M. J.; Trucks, G. W.; Schlegel, H. B.; Scuseria, G. E.; Robb, M. A.; Cheeseman, J. R.; Montgomery, J. A., Jr.; Vreven, T.; Kudin, K. N.; Burant, J. C.; et al. Gaussian 03, Revision E.01; Gaussian, Inc.; Wallingford, CT, 2004.

(38) Soler, J. M.; Artacho, E.; Gale, J. D.; García, A.; Junquera, J.; Ordejón, P.; SánchezPortal, D. The SIESTA Method for Ab Initio Order-N Materials Simulation. J. Phys.: Condens. Matt. 2002, 14, 2745-2779.

(39) Häkkinen, H. The Gold-Sulfur Interface at the Nanoscale. Nature Chem. 2012, 4, 443-455.

(40) Dong, R.; Pfeffermann, M.; Skidin, D.; Wang, F.; Fu, Y.; Narita, A.; Tommasini, M.; Moresco, F.; Cuniberti, G.; Berger, R. et al. Persulfurated Coronene: A New Generation of “Sulflower”. J. Am. Chem. Soc., 2017, 139, 2168-2171.

(41) Movsisyan, L. D.; Peeks, M. D.; Greetham, G. M.; Towrie, M.; Thompson, A. L.; Parker, A. W.; Anderson, H. L. Photophysics of Threaded sp-Carbon Chains: The Polyyne is a Sinkfor Singlet and Triplet Excitation. J. Am. Chem. Soc. 2014, 136, 1799618008.

(42) Agapito, L. A.; Kioussis, N.; Kaxiras, E. Electric-Field Control of Magnetism in Graphene Quantum Dots: Ab Initio Calculations. Phys. Rev. B 2010, 82, 201411.

(43) Dong, Y.-J.; Wang, X.-F.; Zhai, M.-X.; Wu, J.-C.; Zhou, L.; Han, Q.; Wu, X.-M. Effects of Geometry and Symmetry on Electron Transport through Graphene-CarbonChain Junctions. J. Phys. Chem. C 2013, 117, 18845- 18850.

(44) Shen, L.; Zeng, M.; Yang, S.-W.; Zhang, C.; Wang, X.; Feng, Y. Electron Transport Properties of Atomic Carbon Nanowires between Graphene Electrodes. J. Am. Chem. Soc. 2010, 132, 11481-11486. 
(45) Swart, M.; Groenhof, A. R.; Ehlers, A. W.; Lammertsma, K. Validation of ExchangeCorrelation Functionals for Spin States of Iron Complexes. J. Phys. Chem. A 2004, 108, 5479-5483.

(46) Omokawa, Y.; Hatano, S.; Abe, M. Electron-Spin Resonance (ESR) Characterization of Quintet Spin-State Bis-Nitroxide-Bearing Cyclopentane-1,3-Diyl Diradicals. J. Phys. Org. Chem. 2015, 28, 116-120.

(47) Denis, P. A.; Huelmo, C. P.; Martins, A. S. Band Gap Opening in Dual-Doped Monolayer Graphene. J. Phys. Chem. C 2016, 120, 7103-7112.

(48) Denis, P. A. Chemical Reactivity and Band-Gap Opening of Graphene Doped with Gallium, Germanium, Arsenic, and Selenium Atoms. ChemPhysChem 2014, 15, 39944000.

(49) Michael R. Wasielewski, M. R. Organic Semiconductors: Dynamic Duos. Nature Phys. 2017, 13, 114-115.

(50) Han, W.; Kawakami, R. K.; Gmitra, M. Jaroslav Fabian. Graphene Spintronics. Nature Nanotech. 2014, 9, 794-807.

(51) Avsar, A.; Tan, J. Y.; Taychatanapat, T.; Balakrishnan, J.; Koon, G.K.W.; Yeo, Y.; Lahiri, J.; Carvalho, A.; Rodin, A. S.; O’Farrell, E.C.T.; et al. Spin-Orbit Proximity Effect in Graphene. Nature Commun. 2014, 5, 4875.

(52) Chuvilin, A.; Meyer, J. C.; Algara-Siller, G.; Kaiser, U. From Graphene constrictions to single carbon chains. New J. Phys. 2009, 11, 083019.

(53) Pensa, E.; Cortés, E.; Corthey, G.; Carro, P.; Vericat, C.; Fonticelli, M. H.; Benítez, G.; Rubert, A. A.; Salvarezza, R. C. The Chemistry of the Sulfur-Gold Interface: In Search of a Unified Model. Acc. Chem. Res. 2012, 45, 1183-1192. 
(54) Yasuda, S.; Yoshida, S.; Sasaki, J.; Okutsu, Y.; Nakamura, T.; Taninaka, A.; Takeuchi, O.; Shigekawa, H. Bond Fluctuation of S/Se Anchoring Observed in SingleMolecule Conductance Measurements using the Point Contact Method with Scanning Tunneling Microscopy. J. Am. Chem. Soc. 2006, 128, 7746-7747.

(55) Batista, R.; Ordejón, P.; Chacham, H.; Artacho, E. Resistive and Rectifying Effects of Pulling Gold Atoms at Thiol-Gold Nanocontacts. Phys. Rev. B 2007, 75, 041402.

(56) Denis, P. A. Band Gap Opening of Monolayer and Bilayer Graphene Doped with Aluminium, Silicon, Phosphorus, and Sulfur. Chem. Phys. Lett. 2010, 492, 251-257.

(57) Denis, P. A. Density Functional Investigation of Thioepoxidated and Thiolated Graphene. J. Phys. Chem. C 2009, 113, 5612-5619. 


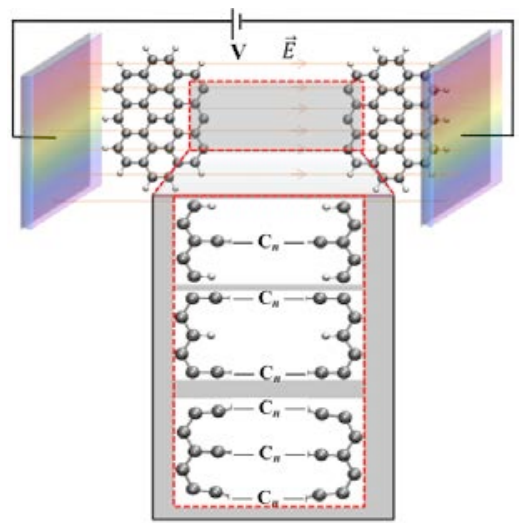

